\title{
Editorial
}

\section{Mathematical and Numerical Modeling of Flow and Transport 2012}

\author{
Shuyu Sun, ${ }^{1}$ Mohamed Fathy El-Amin, ${ }^{1,2}$ \\ Zhangxing Chen, ${ }^{3}$ and Hiroshi Kanayama ${ }^{4}$ \\ ${ }^{1}$ Computational Transport Phenomena Laboratory (CTPL), Division of Physical Sciences and Engineering \\ (PSE), King Abdullah University of Science and Technology (KAUST), Thuwal 23955-6900, Saudi Arabia \\ ${ }^{2}$ Mathematics Department, Faculty of Science, Aswan University, Aswan 81528, Egypt \\ ${ }^{3}$ Schulich School of Engineering, University of Calgary, 2500 University Drive NW, Calgary, \\ $A B$, Canada T2N 1N4 \\ ${ }^{4}$ Department of Mechanical Engineering, Kyushu University, 744 Motooka, Nishi-ku, \\ Fukuoka 819-0395, Japan \\ Correspondence should be addressed to Shuyu Sun, shuyu.sun@kaust.edu.sa \\ Received 18 September 2012; Accepted 18 September 2012 \\ Copyright (C) 2012 Shuyu Sun et al. This is an open access article distributed under the Creative \\ Commons Attribution License, which permits unrestricted use, distribution, and reproduction in \\ any medium, provided the original work is properly cited.
}

\section{Introduction}

Modeling of flow and transport is an essential component of many scientific and engineering applications, with increased interests in recent years. Application areas vary widely and include groundwater contamination, carbon dioxide sequestration, air pollution, petroleum exploration and recovery, weather prediction, and chemical separation processes However, accurate mathematical and numerical simulation of flow and transport remains a challenging topic from many aspects of physical modeling, numerical analysis, and scientific computation. An important step of its numerical solution procedure is to apply advanced discretization methods (e.g., finite elements, finite volumes, and finite differences) to the governing equations. Another important solution step is the design of fast and accurate solvers for the large-scale linear and nonlinear algebraic equation systems that result from discretization. Solution techniques of interest include multiscale algorithms, mesh adaptation, parallel algorithms and implementation, and efficient splitting or decomposition schemes.

\section{Overview of Work Presented in This Annual Issue}

This annual special issue covers a number of developing topics in mathematical modeling and numerical simulations of flow and transport phenomena for a variety of applications. 
The issue effectively blends theoretical, numerical, modeling, and simulation aspects of flow and transport problems that are usually encountered in many research areas. From biological applications to the steel industry, and all the way to nanofluids applications, the 25 papers of this annual issue serve as state-of-the-art important reference for any mathematician or engineer working in a field related to modeling and simulation of flow and transport problems. The topics of this special issue may be divided into eight sections of complex flow and transport: (1) fluid flow problems, (2) flow in porous media, (3) fluid flow and heat transfer, (4) nano- and biofluids, (5) traffic problems, (6) selected problems of industrial challenges, (7) hydrogen safety issues, and (8) integration techniques for flow and transport. We outline each of the eight areas below.

The first group of papers addresses various issues in the area of flow of fluids. A stabilized incompressible smoothed particle hydrodynamics is proposed in the paper " $A$ stabilized incompressible SPH method by relaxing the density invariance condition" to simulate free surface flow. The modification appears in the source term of pressure Poisson equation, and the idea is similar to the recent development in Moving Particle Semi-implicit method (MPS). In a paper entitled "Viscoelastic flow through an axisymmetric contraction using the gridby-grid inversion method," by H. Park, the grid-by-grid inversion algorithm is developed to simulate viscoelastic flow through an axisymmetric contraction. S. Peng has introduced a paper entitled " $1 D$ and $2 D$ numerical modeling for solving dam-break flow problems using finite volume method" to model the flow movement in an idealized dam-break configuration. Onedimensional and two-dimensional motion of a shallow flow over a rigid inclined bed was considered in this study. In the paper "Effects of regional magnetic field on rotating MHD flow field of unity magnetic Prandtl number" S. Hung and J. Leong studied the flow pattern of a magnetic fluid filled within an annulus with moving inner cylinder at a constant rotational speed and stationary outer cylinder under influence of a nonuniform external magnetic field. M. Lee and G. Kim introduced the dispersion coefficient tensor including off-diagonal components of the flow with secondary currents, namely, skewed shear flow dispersion (SSFD) coefficient tensor, in the paper "Influence of secondary currents on solute dispersion in curved open channels."

The second group concerns with flow in porous media. In "FEM analyses for T-H$M-M$ coupling processes in dual-porosity rock mass under stress corrosion and pressure solution" Y. J. Zhang et al. have introduced the models of stress corrosion and pressure solution into the 2D FEM code of thermo-hydro-mechanical-migratory coupling analysis for dualporosity medium. The effects of a porous fence with a nonuniform porosity on flow fields are investigated numerically by L. M. Huang et al. in a paper entitled "A numerical study on flow around nonuniform porous fences." In the paper "Natural convection in an inclined porous cavity with spatial sidewall temperature variations" M. Selamat et al. have studied the natural convection in a porous cavity with a nonuniform hot wall temperature and a uniform cold wall temperature. In the paper "Upscaling of permeability field of fractured rock system: numerical examples" by K. Bao et al., several numerical examples have been considered to calculate effective hydraulic properties for a given fractured porous medium domain. Several scenarios of fractured systems have been considered starting with two fractures up to 800 fractures. T. $\mathrm{H}$. Jung et al. have introduced a numerical experiment in their paper "Characteristics of wave reflection for vertical and slit caissons with porous structures" to investigate the characteristics of a reflected wave from a porous structure located in front of a slit caisson.

Coupling of heat and fluid flow is covered by the third group. S. Almalowi and A. Oztekin presented numerical simulation of two-dimensional convective heat transfer problem using two-dimensional, nine directional D2Q9 thermal lattice Boltzmann arrangements, in the paper entitled "Flow simulations using two-dimensional thermal lattice 
Boltzmann method." In the paper entitled "Thermal diffusion and diffusion thermoeffects on MHD thermosolutal Marangoni convection boundary layer flow over a permeable surface" by R. Hamid et al., the problem of thermal diffusion and diffusion thermoeffects on thermosolutal Marangoni convection flow of an electrically conducting fluid over a permeable surface is investigated. The paper entitled "Airflow and heat transfer in the slot-vented room with radiant floor heating unit" presents numerical simulation of floor radiant heating system with three types of slot ventilation, namely, lateral slot ventilation (LSV), ceiling slot ventilation (CSV) and no slot ventilation (NSV). J. Alinejad and S. Samar bakhsh in their paper, "Viscous flow over nonlinearly stretching sheet with effects of viscous dissipation" have presented an analysis to study the flow and heat transfer phenomenon in a viscous fluid over a nonlinearly stretching sheet by considering the effects of heat dissipation.

The next group of papers is devoted to flow and transport of nanofluids and biofluids. In the paper "Numerical investigation of nanofluid forced convection in channels with discrete heat sources" the flow and heat transfer characteristics of channel flow with discrete heat sources for base fluid (distilled water) and a nanofluid that is composed of distilled water and $\mathrm{Al}_{2} \mathrm{O}_{3}$ nanoparticles are numerically investigated by Mashaei et al. The paper entitled "Nonlinear fluid models for biofluid flow in constricted blood vessels under body accelerations: a comparative study" by D. Sankar and A. Nagar presents a mathematical analysis that represents various interesting rheological properties of blood when it flows through narrow stenosed arteries with body acceleration, treating it as different non-Newtonian fluid models with yield stress such as Herschel-Bulkley fluid model and Casson fluid model.

The fifth group concerns with some traffic problems. The paper "Evaluation of congestion relief proposals in a capital city" aims at analyzing three different solutions suggested for traffic congestion relief in Port Louis, the busiest city of Mauritius. Three alternatives use light rail transit (LRT) as an alternative mode of transport, the construction of a ring road around Port Louis, and the upgrading of the current bus network into a bus rapid transit (BRT) system. Assuming that platoon speed follows a truncated normal distribution, ranging from minimum speed to maximum speed, the paper "A platoon dispersion model based on a truncated normal distribution of speed" by M. Wei et al. develops a piecewise density function that describes platoon dispersion characteristics as the platoon moves from an upstream to a downstream intersection.

Selected problems from industry have been covered in the sixth group. The objective of the paper "Mathematical analysis of inclusion removal from liquid steel by gas bubbling in a casting tundish" is to analyze and improve the understanding of the alumina inclusion removal rate by bubble attachment and by gas bubbling fluid dynamics effects. In a paper entitled "Numerical simulation of oil spill in ocean," a numerical model is employed to solve a two-dimensional advection-diffusion-reaction equation. The model is based on a standard split operator (fractional step) approach. N. Leitão has introduced a paper entitled "GMM estimator: an application to intraindustry trade" to investigate the determinants of intraindustry trade (IIT), horizontal IIT (HIIT), and vertical IIT (VIIT) in the automobile industry in Portugal. The GMM system is applied to solve the problems of serial correlation and the endogeneity of some explanatory variables.

The seventh group is devoted to hydrogen safety issues. In the paper "Theoretical analysis and semianalytical solutions for a turbulent buoyant hydrogen-air jet" semianalytical solutions are developed by M. El-Amin et al. for turbulent hydrogen-air plume. Analytical expressions are derived for plume centerline variables (radius, velocity, and density deficit) in terms of a single universal function. I. Ismail et al. have investigated the effect of wind speed and tunnel geometry on the flow and dispersion of hydrogen within tunnel structures 
in a paper entitled "Modelling considerations in the simulation of hydrogen dispersion within tunnel structures."

Finally, in the eighth group some integration techniques for flow and transport are introduced. In the paper "The technique of MIEELDLD in computational aeroacoustics," Appadu has used the technique of minimised integrated exponential error for low dispersion and Low Dissipation (MIEELDLD) in a computational aeroacoustics framework to obtain modifications to optimized spatial schemes constructed in a previous work. A new method for solving nonlinear Volterra-Fredholm-Hammerstein (VFH) integral equations is presented in the paper "A new direct method for solving nonlinear Volterra-Fredholm-Hammerstein integral equations via optimal control problem." The method is based on reformulation of VFH to the simple form of Fredholm integral equations and hence converting it to optimal control problem.

\section{Conclusions}

Improvement of computer hardware and numerical algorithms in recent years had made more accurate and higher-resolution simulations of flow and transport possible, which yielded a better and deeper understanding of flow and transport and their interaction with other physical, chemical, biological, and sociological processes. This special issue focuses on eight important areas of flow and transport and it highlights new applications and new challenges. Like the previous issue, this issue is not intended to be an exhaustive collection nor a survey of all of the current trends in flow and transport research; many additional significant research areas of flow and transport still exist and remain to be explored, but multidisciplinary research effort is a clear trend.

\section{Acknowledgments}

The authors would like to thank the participants of the special issue for their inspiring contributions, and the anonymous reviewers for their diligent work, which led to the high quality of the special issue. The lead guest editor S. Sun would like to acknowledge KAUST Faculty Baseline Research Fund (BRF) for supporting his research in flow and transport.

Shuyu Sun

Mohamed Fathy El-Amin

Zhangxing Chen

Hiroshi Kanayama 


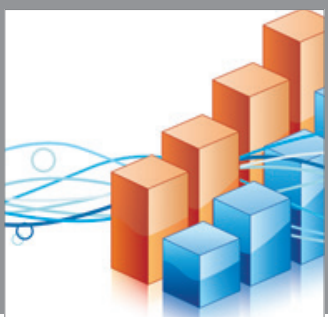

Advances in

Operations Research

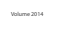

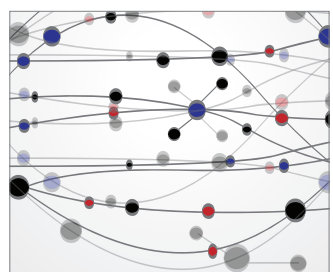

\section{The Scientific} World Journal
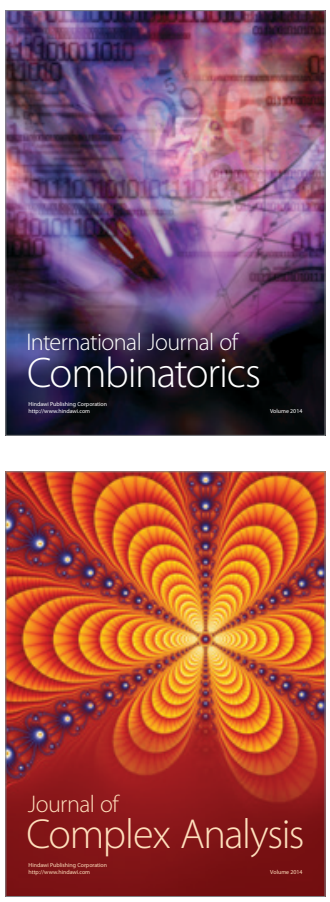

International Journal of

Mathematics and

Mathematical

Sciences
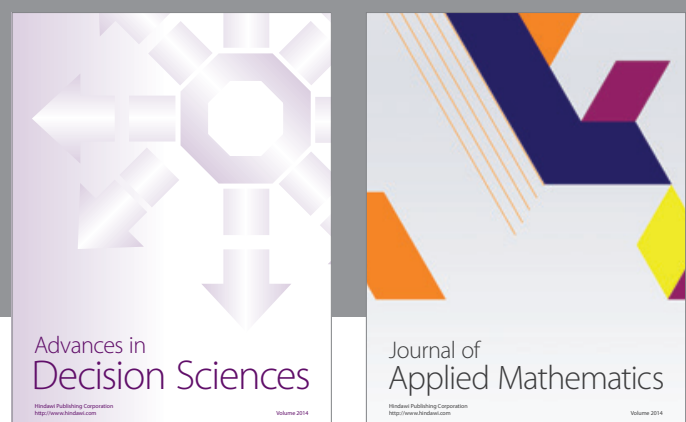

Journal of

Applied Mathematics
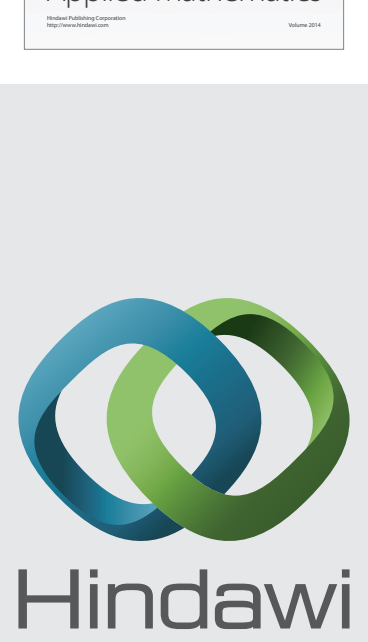

Submit your manuscripts at http://www.hindawi.com
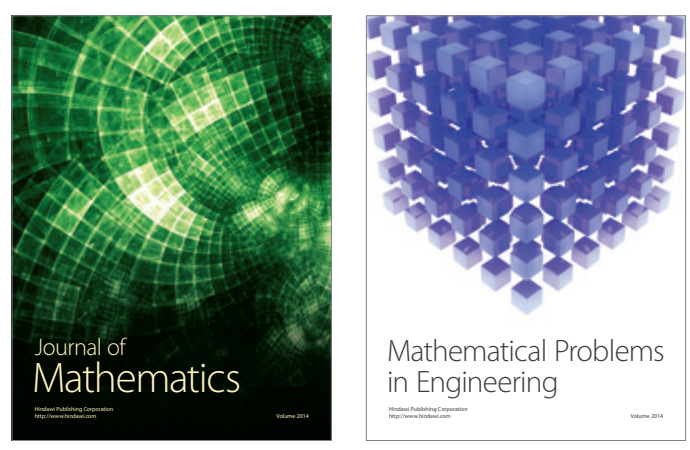

Mathematical Problems in Engineering
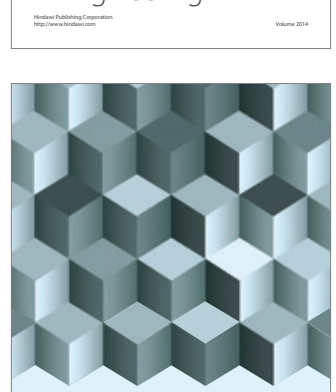

Journal of

Function Spaces
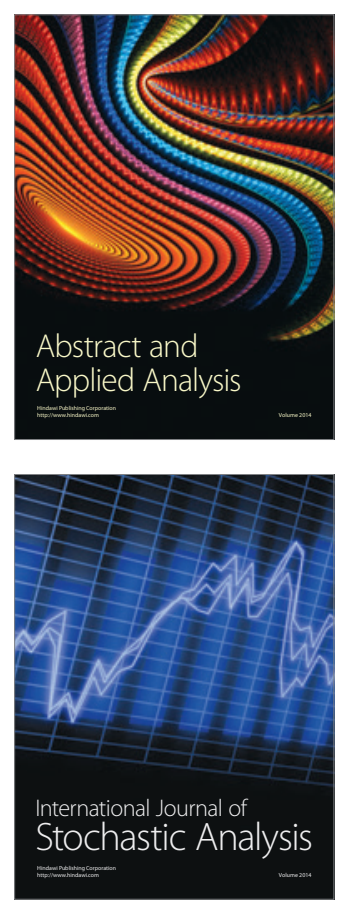

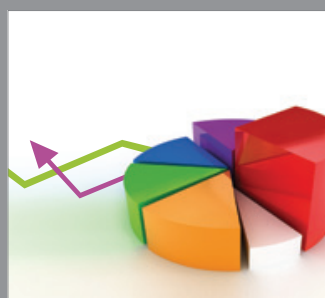

ournal of

Probability and Statistics

Promensencen
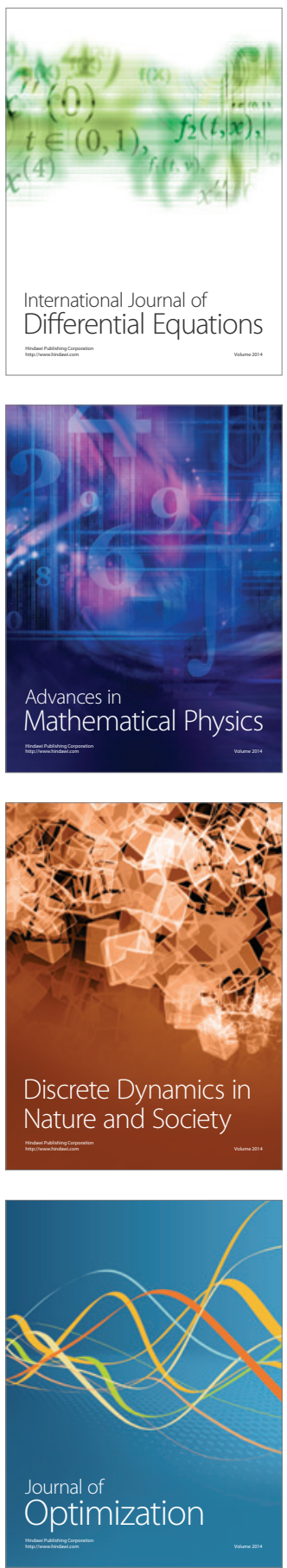